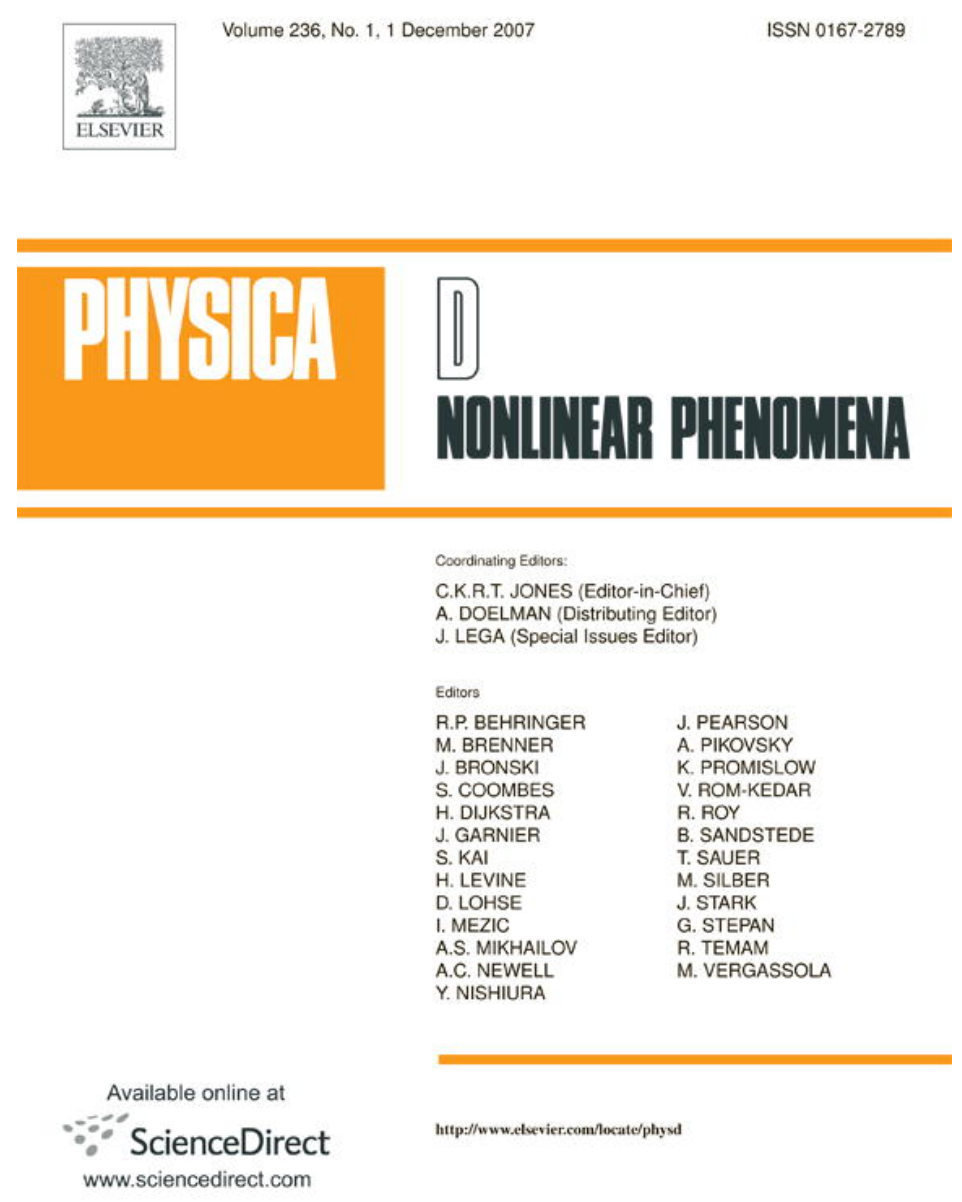

This article was published in an Elsevier journal. The attached copy

is furnished to the author for non-commercial research and education use, including for instruction at the author's institution, sharing with colleagues and providing to institution administration.

Other uses, including reproduction and distribution, or selling or licensing copies, or posting to personal, institutional or third party websites are prohibited.

In most cases authors are permitted to post their version of the article (e.g. in Word or Tex form) to their personal website or institutional repository. Authors requiring further information regarding Elsevier's archiving and manuscript policies are encouraged to visit:

http://www.elsevier.com/copyright 


\title{
Well-posedness for solid-liquid phase transitions with a fourth-order nonlinearity
}

\author{
V. Berti ${ }^{a, *}$, M. Fabrizio ${ }^{a, 1}$, C. Giorgi ${ }^{b, 2}$ \\ a Dipartimento di Matematica, Università di Bologna, Piazza di Porta S. Donato 5, 40126 Bologna, Italy \\ ${ }^{\mathrm{b}}$ Dipartimento di Matematica, Università di Brescia, Via Valotti 9, 25133 Brescia, Italy
}

Received 20 October 2006; received in revised form 5 February 2007; accepted 13 July 2007

Available online 18 July 2007

Communicated by R. Temam

\begin{abstract}
A phase-field system which describes the evolution of both the absolute temperature $\theta$ and the phase variable $f$ during first-order transitions in thermal insulators is considered. A thermodynamic approach is developed by regarding the order parameter as a phase field and its evolution equation as a balance law. By virtue of the special form of the internal energy, a third-order nonlinearity $G_{2}^{\prime}(f)$ appears in the energy balance in place of the (customarily constant) latent heat. As a consequence, the bounds $0 \leq f \leq 1$ hold whenever $\theta$ is positive valued. In addition, a nonlinear Fourier law with conductivity proportional to temperature is assumed. Well-posedness for the resulting initial and boundary value problem are then established in a suitable setting.
\end{abstract}

(C) 2007 Elsevier B.V. All rights reserved.

Keywords: Phase transition; Phase-field models; Continuum thermodynamics; Existence and uniqueness

\section{Introduction}

In this paper we investigate the well-posedness of a phase-field model describing temperature-induced solid-liquid transitions in materials whose thermal conductivity increases linearly with the temperature. The full model is developed in Section 2, regarding the order parameter $f$ of the transition as a phase field $f(x, t)$ which changes smoothly in the domain $\Omega \subset \mathbb{R}^{3}$. As is customary, $f$ represents the solid concentration, and $f=0$ for the fluid phase, $f=1$ for the solid phase. In the cylinder $\Omega \times(0, T)$ the evolution of the phase field $f(x, t)$ and the absolute temperature $\theta(x, t)$ is ruled by the following system:

$f_{t}-\kappa \Delta f+G_{1}^{\prime}(f)+\frac{\theta}{\theta_{c}} G_{2}^{\prime}(f)=0$

\footnotetext{
* Corresponding author. Tel.: +39 51 2094415; fax: +39 512094490.

E-mail addresses: berti@dm.unibo.it (V. Berti), fabrizio@dm.unibo.it

(M. Fabrizio), giorgi@ing.unibs.it (C. Giorgi).

1 Tel.: +39 51 2094444; fax: +39512094490.

2 Tel.: +39303715778.
}

$\partial_{t}\left(\alpha(\theta)-\frac{1}{\theta_{c}} G_{2}(f)\right)-k_{0} \Delta \theta=R$

endowed with initial and Neumann-Dirichlet boundary conditions. Here and in the sequel $\partial_{t}$, as well as the subscript ${ }_{t}$, denote differentiation with respect to time.

The kinetic equation governing the phase-field evolution is derived here from a balance law along the lines of [9, 10]. It takes the form of the non-isothermal Ginzburg-Landau equation (1.1), where $\theta_{c}$ is the temperature transition value (see [13]). As is customary in first-order transitions, the function $G=G_{1}+G_{2}$ provides the so-called "double-well potential" which has two wells of equal depth located at $f=0$ and $f=1$. When $\theta=\theta_{c}$, only two bulk (i.e. stationary and uniform) stable equilibrium solutions to (1.1) exist: $f(x, t)=0$ and $f(x, t)=1$.

In order to derive (1.2), a nonlinear Fourier heat conduction law with conductivity proportional to temperature is assumed, namely

$\mathbf{q}(t)=-k_{0} \theta(t) \nabla \theta(t)$. 
This is the case when insulating media are considered (see [17]). In water, for instance, the heat transfer is mainly due to convection, so ice looks like a thermal insulator (the igloo is based on this property) and the same holds true for water at rest. To check whether this system is in agreement with thermodynamics, we define the second law by means of the Clausius-Duhem inequality. Accordingly, we take a suitable choice of the free energy density $\psi$ and, after multiplying by $1 / \theta$ and neglecting some terms, the energy balance equation yields (1.2) with $\alpha(\theta)=\ln \theta$.

Phase-field models like (1.1) and (1.2) have been extensively studied (see, for instance, $[6,12]$ and references therein) and applied to realistic materials problems [14]. In the standard Caginalp model [7], $\alpha$ and $G_{2}$ are linear in $\theta$ and $f$, respectively. Penrose and Fife in [20] proposed a different class of models where $G_{2}$ has (at most) quadratic growth. Some solidification models (see, for instance, [22,23]) assume that $G_{2}$ has degree 3 or 5. Finally, in [8] a cubic nonlinearity $G_{2}$ is scrutinized in order to model superheating and undercooling.

According to [14], the choice of $G_{2}$ with an odd degree is motivated by making more efficient numerical simulations in the small undercooling regime. Nevertheless, there is no reason to rule out $G_{2}$ being even. Thus, following [9], we assume $G_{1}$, $G_{2}$ to be fourth-order polynomials such that

$G_{1}(f)+G_{2}(f)=G(f)=6 L f^{2}(1-f)^{2}$

and the following properties of solutions to (1.1) hold:

P. $1-f(x, t)=0$ and $f(x, t)=1$ correspond to bulk solid and liquid equilibrium states for all values of the temperature $\theta$,

P. 2 - at $\theta=\theta_{c}$ both states are stable, and a change of stability occurs when $\theta<\theta_{c}$ or $\theta>\theta_{c}$,

P.3 - superheating and undercooling are allowed when $\theta$ lies in a neighborhood of $\theta_{c}$.

It is worth noting that P.1 cannot be satisfied when the polynomial degree of $G_{1}^{\prime}$ and $G_{2}^{\prime}$ is lower than 2. Thus, neither Caginalp nor Penrose-Fife models agree with P.1. Assuming (1.3), if $G_{2}$ is a cubic nonlinearity then the validity of P.2 and P.3 is restricted to a suitable neighborhood of $\theta_{c}$, but no restriction occurs when $G_{2}$ has degree 5 [22]. As newly remarked in [9], properties P.1-P.3 are fulfilled for any temperature even when $G_{1}$ and $G_{2}$ are suitable fourth-order functions, namely

$G_{1}(f)=L\left(3 f^{4}-4 f^{3}\right)$ and

$G_{2}(f)=L\left(3 f^{4}-8 f^{3}+6 f^{2}\right)$.

What is more, this choice enables one to prove the boundedness of $f$ (see Proposition 2.1),

$0 \leq f(x, t) \leq 1$,

provided that $0 \leq f(x, 0) \leq 1$ and $\theta(x, t)>0$ for all $t>0$.

Well-posedness of the initial-boundary value problem generated by (1.1) and (1.2) is proved here by means of the following procedure. First we construct a sequence $G_{2}^{\varepsilon}$ of functions with Lipschitz derivative, approaching $G_{2}$ and behaving as well as $G_{2}$ in $(0,1)$. Then, exploiting a recent result of Colli et al. [2], we obtain existence, uniqueness and positivity of the temperature when $G_{2}^{\varepsilon}$ is considered in place of $G_{2}$. Even in this case P.1 is fulfilled. Then Proposition 2.1 applies and ensures that (1.4) holds for all $\varepsilon$. Finally, we pass to the limit as $\varepsilon \rightarrow 0$ and prove both uniform convergence and uniqueness of solutions via energy methods. The novelty of our result consists in obtaining the boundedness of the phasefield solution directly in the customary setting of a double-well potential, with no recourse to the interval indicator function and its subdifferential, which are rather typical of the Stefan problem with a sharp interface.

Assuming $\alpha(\theta)=c \theta$ and $G_{2}(f)=L f$, problems like (1.1) and (1.2) were studied first in [7] and well-posedness has been established. There, $\theta$ stands for the variation of the temperature relative to the critical value, and its positivity is not expected. In addition, in that model the boundedness of $f$ is not implied. Hence, in order to force the phase variable $f$ to sit between 0 and $1, G_{1}$ is assumed to be - or include - the indicator function $I$ of the interval $(0,1)$ (see, for instance, [16]). This is the case when a "mushy region" does exist at $\theta=\theta_{c}$, and all possible values $f \in[0,1]$ are allowed at equilibrium, not only $f=0$, $f=1$. Regarding the long time behavior and existence of a maximal attractor for phase-field systems of this kind, the reader is referred to, e.g., [15,21].

When $\alpha(\theta)=c \theta$ and $G_{2}(f)$ is a quadratic nonlinearity, phase-field systems like (1.1) and (1.2) are also useful for describing ferromagnetic transformations (see, e.g., [20]). Wellposedness and long time behavior results were proved first in [18] via energy methods. Neither positivity of $\theta$ nor boundedness of $f$ are established.

Recently, in [2,3] a thorough investigation was carried out for a more general model with thermal memory. In particular, when $\alpha(\theta)=\ln \theta, G_{2}(f)=L f$ and $G_{1}=I$, the indicator function of the interval $(0,1)$, existence and uniqueness have been shown jointly with positivity of the temperature field.

The plan of the paper is as follows. In Section 2, we formulate the model in a proper thermodynamic setting and prove Proposition 2.1. In Section 3 we obtain the existence of one solution with positive temperature and bounded phase field. Finally, in Section 4 we state uniqueness and continuous dependence results.

\section{Phase transition model}

Let us consider a material occupying a bounded domain $\Omega$ of $\mathbb{R}^{3}$ whose boundary $\partial \Omega$ has the unit normal vector $\mathbf{n}$. We assume that the mass density $\rho$ is constant and for the sake of simplicity we let $\rho=1$. The state of the material is described by the order parameter $f$, which represents the concentration of the solid phase, and by the absolute temperature $\theta$.

The evolution equation for the phase field is deduced from the balance of the structure order, expressed through the equation

$\int_{S} k \mathrm{~d} x=\int_{\partial S} \mathbf{p} \cdot \mathbf{n}_{S} \mathrm{~d} a+\int_{S} \sigma \mathrm{d} x$ 
for any smooth subbody $S \subset \Omega$ with boundary $\partial S$ and unit normal vector $\mathbf{n}_{S}$. Here $k$ is the internal structure order density, $\mathbf{p}$ and $\sigma$ are the structure order flux and supply. By applying the divergence theorem to (2.5), we obtain the local formulation

$k=\nabla \cdot \mathbf{p}+\sigma$.

In particular, in order to describe a first-order phase transition, we consider the constitutive equations

$k=f_{t}+g_{1}(f)+\frac{\theta}{\theta_{c}} g_{2}(f)$

$\mathbf{p}=\kappa \nabla f$,

where $\theta_{c}$ is the critical temperature of the phase transition, $\kappa$ is a positive constant, $g_{1}, g_{2}$ are two functions depending on the phase field $f$. Moreover we assume $\sigma=0$.

Substitution into (2.6) leads to the differential equation

$f_{t}+g_{1}(f)+\frac{\theta}{\theta_{c}} g_{2}(f)=\kappa \Delta f$.

The evolution equation for the temperature can be obtained from the energy balance law, written in the local form as

$h=-\nabla \cdot \mathbf{q}+r$

where $h$ is the rate at which heat is absorbed, $\mathbf{q}$ is the heat flux vector and $r$ is the heat supply. Moreover, in view of the first law of thermodynamics

$e_{t}=\mathcal{P}^{i}+h$,

where $e$ is the internal energy and $\mathcal{P}^{i}$ denotes the internal action power. Therefore

$e_{t}=\mathcal{P}^{i}-\nabla \cdot \mathbf{q}+r$.

In this model we neglect the internal mechanical power due to macroscopic movements and assume that $\mathcal{P}^{i}$ is the internal structure order power, defined as

$$
\begin{aligned}
\mathcal{P}^{i}=k f_{t}+\mathbf{p} \cdot \nabla f_{t}= & f_{t}^{2}+\partial_{t} G_{1}(f)+\frac{\theta}{\theta_{c}} \partial_{t} G_{2}(f) \\
& +\frac{\kappa}{2} \partial_{t}\left(|\nabla f|^{2}\right),
\end{aligned}
$$

with $G_{1}$ and $G_{2}$ such that $G_{1}^{\prime}(f)=g_{1}(f), G_{2}^{\prime}(f)=g_{2}(f)$, $G_{1}(0)=G_{2}(0)=0$. Therefore Eq. (2.10) reads

$\partial_{t}\left[e-G_{1}(f)-\frac{\kappa}{2}|\nabla f|^{2}\right]-\frac{\theta}{\theta_{c}} \partial_{t} G_{2}(f)-f_{t}^{2}$

$$
=-\nabla \cdot \mathbf{q}+r \text {. }
$$

The constitutive law for the heat flux is usually given by

$\mathbf{q}=-k(\theta) \nabla \theta$,

where $k(\theta)$ is the thermal conductivity. When insulating materials, for instance ice and water, are considered, the conductivity is allowed to depend linearly on the temperature [4,17], namely

$\mathbf{q}=-k_{0} \theta \nabla \theta$, with $k_{0}>0$. Therefore Eq. (2.12) can be written as

$$
\begin{aligned}
\partial_{t} & {\left[e-G_{1}(f)-\frac{\kappa}{2}|\nabla f|^{2}\right]-\frac{\theta}{\theta_{c}} \partial_{t} G_{2}(f)-f_{t}^{2} } \\
& =k_{0} \theta \Delta \theta+k_{0}|\nabla \theta|^{2}+r .
\end{aligned}
$$

Now, we prove that this model is compatible with the second law of thermodynamics, expressed in terms of the Clausius-Duhem inequality [11]

$\eta_{t} \geq-\nabla \cdot\left(\frac{\mathbf{q}}{\theta}\right)+\frac{r}{\theta}$

where $\eta$ is the entropy. Relations (2.10), (2.11) and (2.15) imply $\theta \eta_{t} \geq e_{t}-k f_{t}-\mathbf{p} \cdot \nabla f_{t}+\frac{1}{\theta} \mathbf{q} \cdot \nabla \theta$.

By introducing the free energy $\psi=e-\theta \eta$, in view of the representations (2.7) and (2.8), we have

$$
\begin{aligned}
\psi_{t} & +\eta \theta_{t}-f_{t}^{2}-\left[g_{1}(f)+\frac{\theta}{\theta_{c}} g_{2}(f)\right] f_{t}-\kappa \nabla f \cdot \nabla f_{t} \\
& +\frac{1}{\theta} \mathbf{q} \cdot \nabla \theta \leq 0 .
\end{aligned}
$$

Finally, from (2.13) we obtain the inequality

$$
\begin{aligned}
\psi_{t} \leq & -\eta \theta_{t}+f_{t}^{2}+\left[g_{1}(f)+\frac{\theta}{\theta_{c}} g_{2}(f)\right] f_{t}+\kappa \nabla f \cdot \nabla f_{t} \\
& +k_{0}|\nabla \theta|^{2} .
\end{aligned}
$$

By assuming $\psi=\psi(\theta, f, \nabla f)$, from the arbitrariness of $\theta_{t}, f_{t}, \nabla f_{t}$, we deduce

$\eta=-\frac{\partial \psi}{\partial \theta}, \quad g_{1}(f)+\frac{\theta}{\theta_{c}} g_{2}(f)=\frac{\partial \psi}{\partial f}, \quad \kappa \nabla f=\frac{\partial \psi}{\partial \nabla f}$.

Hence

$\psi(\theta, f, \nabla f)=-C(\theta)+G_{1}(f)+\frac{\theta}{\theta_{c}} G_{2}(f)+\frac{\kappa}{2}|\nabla f|^{2}$

$\eta(\theta, f)=c(\theta)-\frac{1}{\theta_{c}} G_{2}(f)$,

where $C^{\prime}(\theta)=c(\theta)$. Substitution into (2.16) leads to the reduced inequality

$0 \leq f_{t}^{2}+k_{0}|\nabla \theta|^{2}$

which holds true and proves the thermodynamical consistency of the model.

In order to specialize the system of differential equations describing the evolution of this first-order phase-field model, we choose

$c(\theta)=\alpha_{0}(1+\ln \theta)$,

with $\alpha_{0}>0$, and

$g_{1}(f)=12 L f^{2}(f-1), \quad g_{2}(f)=12 L f(1-f)^{2}$,

with $L>0$. Accordingly

$G_{1}(f)=L\left(3 f^{4}-4 f^{3}\right), \quad G_{2}(f)=L\left(3 f^{4}-8 f^{3}+6 f^{2}\right)$ 
and

$$
\begin{aligned}
& \psi=-\alpha_{0} \theta \ln \theta+L\left(3 f^{4}-4 f^{3}\right)+\frac{L}{\theta_{c}}\left(3 f^{4}-8 f^{3}+6 f^{2}\right) \theta \\
& +\frac{\kappa}{2}|\nabla f|^{2} \\
& \eta=\alpha_{0}(1+\ln \theta)-\frac{L}{\theta_{c}}\left(3 f^{4}-8 f^{3}+6 f^{2}\right) \\
& e=\psi+\theta \eta=\alpha_{0} \theta+L\left(3 f^{4}-4 f^{3}\right)+\frac{\kappa}{2}|\nabla f|^{2} .
\end{aligned}
$$

In view of these choices, at the transition temperature $\theta=$ $\theta_{c}$, the free energy depends on the phase field $f$ through the term

$L\left(3 f^{4}-4 f^{3}\right)+L\left(3 f^{4}-8 f^{3}+6 f^{2}\right) \frac{\theta}{\theta_{c}}=6 L f^{2}(1-f)^{2}$,

which coincides with the usual double-well potential. Moreover, since

$\theta_{c}\left[\eta\left(\theta_{c}, 0\right)-\eta\left(\theta_{c}, 1\right)\right]=L$,

the constant $L$ can be identified with the latent heat absorbed during the first-order transition.

In view of (2.19) and (2.22), the heat equation (2.14) takes the form

$\alpha_{0} \theta_{t}-\frac{12 L}{\theta_{c}} \theta f(1-f)^{2} f_{t}-f_{t}^{2}=k_{0} \theta \Delta \theta+k_{0}|\nabla \theta|^{2}+r$.

Hereafter, we consider the approximation of this equation obtained by neglecting the terms proportional to $f_{t}^{2}$ and $|\nabla \theta|^{2}$, namely

$\alpha_{0} \theta_{t}-\frac{12 L}{\theta_{c}} \theta f(1-f)^{2} f_{t}=k_{0} \theta \Delta \theta+r$.

This means that the reduced inequality (2.17) holds as an equality, and the thermodynamical consistency is still ensured. By multiplying (2.23) by $\frac{1}{\theta}$, we finally obtain

$\alpha_{0}(\ln \theta)_{t}-\frac{12 L}{\theta_{c}} f(1-f)^{2} f_{t}=k_{0} \Delta \theta+\frac{r}{\theta}$.

In the sequel we restrict our attention to special situations in which either $r=0$, or the temperature $\theta$ remains very close to the transition temperature $\theta_{c}$. If this is the case, the source $\frac{r}{\theta}$ can be approximated by

$\frac{r}{\theta} \approx r\left[\frac{1}{\theta_{c}}-\frac{1}{\theta_{c}^{2}}\left(\theta-\theta_{c}\right)\right]$

and this in turn implies the further approximation

$\alpha_{0}(\ln \theta)_{t}-\frac{12 L}{\theta_{c}} f(1-f)^{2} f_{t}=k_{0} \Delta \theta+\frac{r}{\theta_{c}}\left(2-\frac{\theta}{\theta_{c}}\right)$.

In view of (2.18), the phase-field evolution equation (2.9) can be written in the form

$f_{t}=\kappa \Delta f+12 L f^{2}(1-f)-12 L \frac{\theta}{\theta_{c}} f(1-f)^{2}$.
The differential equations (2.24) and (2.25) are completed with a non-homogeneous Dirichlet condition for the temperature and a Neumann boundary condition for the phase field, namely

$\left.\theta\right|_{\partial \Omega}=\theta_{\Gamma}$

$\left.\nabla f \cdot \mathbf{n}\right|_{\partial \Omega}=0$.

As a preliminary result, we scrutinize Eq. (2.25) with boundary condition (2.27) disregarding the heat equation (2.24) in the system and assuming that $\theta$ is a given nonnegative field. In this case we state that (2.25) and (2.27) lead to the boundedness of the phase field. The proof is given in [9], where a different system involving the same equation is considered.

Proposition 2.1. Given $\theta \geq 0$ in $\Omega \times(0, T)$, if $f$ is a solution to (2.25) and (2.27) with initial datum $f(x, 0)=f_{0}(x)$ satisfying $0 \leq f_{0} \leq 1$ a.e. in $\Omega$, then

$0 \leq f \leq 1, \quad$ a.e. in $\Omega \times(0, T)$.

Remark 2.1. The boundedness of $f$ can be proved even if we replace $g_{2}(f)=12 L f(1-f)^{2}$ by $\mu(f)$, where $\mu$ is a continuous function such that $z \mu(z) \geq 0$, for all $z \in \mathbb{R}$.

\section{Existence of solutions}

First, we introduce some notation. For any given Hilbert space $\mathcal{H}$ let $\langle\cdot, \cdot\rangle_{\mathcal{H}}$ and $\|\cdot\|_{\mathcal{H}}$ denote the $\mathcal{H}$-inner product and $\mathcal{H}$-norm, respectively. By analogy, the norm of any Banach space $\mathcal{B}$ is denoted by $\|\cdot\|_{\mathcal{B}}$. In particular, let $\langle\cdot, \cdot\rangle$ and $\|\cdot\|$ denote the inner product and norm in $L^{2}(\Omega)$. Sometimes, $\langle\cdot, \cdot\rangle$ stands for the duality pairing between $H_{0}^{1}(\Omega)$ and $H^{-1}(\Omega)$ and $\langle\langle\cdot, \cdot\rangle\rangle$ denotes the duality pairing between $L^{2}\left(0, T, H_{0}^{1}(\Omega)\right)$ and $L^{2}\left(0, T, H^{-1}(\Omega)\right)$.

We recall that for every $v \in H_{0}^{1}(\Omega)$ the Poincaré inequality

$\lambda_{0}(\Omega)\|v\|^{2} \leq\|\nabla v\|^{2}$

holds. If $v \in H^{2}(\Omega) \cap H_{0}^{1}(\Omega)$, the Poincaré and Young inequalities yield

$\gamma_{0}(\Omega)\|\nabla v\|^{2} \leq\|\Delta v\|^{2}$

In this section we prove the existence of a solution to the problem

$$
P)\left\{\begin{array}{l}
f_{t}=\kappa \Delta f+12 L f^{2}(1-f)-\frac{12 L}{\theta_{c}} \theta f(1-f)^{2} \\
\alpha_{0}(\ln \theta)_{t}-\frac{12 L}{\theta_{c}} f(1-f)^{2} f_{t}=k_{0} \Delta \theta+\frac{r}{\theta_{c}}\left(2-\frac{\theta}{\theta_{c}}\right) \\
\left.\nabla f \cdot \mathbf{n}\right|_{\partial \Omega}=0,\left.\quad \theta\right|_{\partial \Omega}=\theta_{\Gamma} \\
f(x, 0)=f_{0}(x), \quad \theta(x, 0)=\theta_{0} .
\end{array}\right.
$$

For simplicity we suppose $\theta_{\Gamma}$ independent of $t$. Moreover for each $t \in[0, T]$, we define $Q_{t}=\Omega \times(0, t)$ and assume

(H1) $r \in L^{\infty}\left(Q_{T}\right), r_{t} \in L^{\infty}\left(Q_{T}\right)$;

(H2) $f_{0} \in H^{1}(\Omega), 0 \leq f_{0} \leq 1$ a.e. in $\Omega, \theta_{0} \in L^{\infty}(\Omega)$;

(H3) $\theta_{\Gamma} \in H^{1 / 2}(\partial \Omega) \cap L^{\infty}(\partial \Omega)$; 
(H4) there exist two positive constants $\theta_{*}$ and $\theta^{*}$ such that $\theta_{*} \leq \theta_{\Gamma} \leq \theta^{*}, \theta_{*} \leq \theta_{0} \leq \theta^{*}$.

In order to deal with homogeneous boundary conditions we introduce the function $\theta_{\mathcal{H}} \in H^{1}(\Omega)$, which solves the problem

$\left\{\begin{array}{l}\Delta \theta_{\mathcal{H}}=0, \quad \text { in } \Omega \\ \left.\theta_{\mathcal{H}}\right|_{\partial} \Omega=\theta_{\Gamma}\end{array}\right.$

Accordingly, the following inequalities hold:

$\theta_{*} \leq \theta_{\mathcal{H}} \leq \theta^{*}, \quad$ a.e. in $\Omega$,

$\left\|\theta_{\mathcal{H}}\right\|_{H^{1}(\Omega)} \leq c\left\|\theta_{\Gamma}\right\|_{H^{1 / 2}(\partial \Omega)}$.

Henceforth, we denote by $u$ the difference

$u=\theta-\theta_{\mathcal{H}}$

which satisfies a homogeneous Dirichlet boundary condition. Therefore, the Poincaré inequality holds and gives

$\|u\| \leq\|u\|_{H^{1}(\Omega)} \leq C_{\Omega}\|\nabla u\|, \quad C_{\Omega}=\frac{1}{\lambda_{0}(\Omega)}$.

Finally, we assume the following lower bound on the source $r$ :

(H5) $\inf _{Q_{T}} r=b \geq-\frac{k_{0} \theta_{c}^{2}}{C_{\Omega}}$.

\subsection{An auxiliary problem}

In order to prove the existence of solutions to problem $(P)$, we introduce the auxiliary problem

$\left(P^{\prime}\right) \quad\left\{\begin{array}{l}f_{t}=\kappa \Delta f+12 L f^{2}(1-f)-\lambda^{\prime}(f) \frac{\theta}{\theta_{c}} \\ \alpha_{0}(\ln \theta)_{t}-\frac{1}{\theta_{c}} \lambda^{\prime}(f) f_{t}=k_{0} \Delta \theta+\frac{r}{\theta_{c}}\left(2-\frac{\theta}{\theta_{c}}\right) \\ \left.\nabla f \cdot \mathbf{n}\right|_{\partial \Omega}=0,\left.\quad \theta\right|_{\partial \Omega}=\theta_{\Gamma} \\ f(x, 0)=f_{0}(x), \quad \theta(x, 0)=\theta_{0}\end{array}\right.$

with $\lambda \in C^{1}(\mathbb{R})$ and $\lambda^{\prime}$ Lipschitz continuous.

The existence of solutions to problem $\left(P^{\prime}\right)$ can be proved with the same technique as was used in [2, Theorem 2.1]. The main difference here concerns the first equation of $\left(P^{\prime}\right)$, where the term $12 L f^{2}(1-f)$ appears in place of the sum $-\sigma^{\prime}(f)-$ $\beta(f)$. Since $\sigma^{\prime}$ and $\beta$ are assumed to be a Lipschitz continuous function and a maximal monotone graph, respectively, the first equation of $\left(P^{\prime}\right)$ reads as a special case of (2.21) in [2] by means of the following identifications:

$\beta(f)=f^{3}-f^{2}+v f, \quad \sigma^{\prime}(f)=-v f$,

where $v>\frac{1}{3}$ in order to guarantee the monotonicity of $\beta$. Assuming that the memory kernel vanishes, the integrodifferential equation (2.20) of [2] reduces to the second equation of $\left(P^{\prime}\right)$ except for the last term on the right-hand side, i.e. $r \theta / \theta_{c}^{2}$. Nevertheless, this additional term is linear in $\theta$ and, in view of (H1), (H5), the following existence result can be achieved as in [2] with minor modifications.

Theorem 3.1. Problem $\left(P^{\prime}\right)$ admits at least a solution $(f, \theta)$ such that

$f \in L^{2}\left(0, T, H^{2}(\Omega)\right) \cap H^{1}\left(0, T, L^{2}(\Omega)\right)$, $\theta \in L^{2}\left(0, T, H^{1}(\Omega)\right), \quad \theta>0$ a.e. in $Q_{T}$,

$\ln \theta \in L^{\infty}\left(0, T, L^{2}(\Omega)\right) \cap H^{1}\left(0, T, H^{-1}(\Omega)\right)$.

\subsection{Approximating problems}

In order to solve problem $(P)$, we construct here a family of approximating problems of type $\left(P^{\prime}\right)$. To this end, following the Yosida regularization scheme, for each $\varepsilon \in(0,1]$, we consider the function

$\varphi_{\varepsilon}(z)=z+\varepsilon g_{2}(z), \quad z \in \mathbb{R}$,

where $g_{2}$ is defined by (2.18).

Taking into account that $\varphi_{\varepsilon}$ is invertible and letting $J_{\varepsilon}=$ $\varphi_{\varepsilon}^{-1}$, we introduce the function

$\mu_{\varepsilon}(z)=\frac{z-J_{\varepsilon}(z)}{\varepsilon}$.

It is easy to check that $\mu_{\varepsilon}: \mathbb{R} \rightarrow \mathbb{R}$ satisfies the following properties (see [5]):

- $\mu_{\varepsilon}$ is $C^{1}(\mathbb{R})$ and Lipschitz continuous for all $\varepsilon \in(0,1]$,

- $\mu_{\varepsilon}(z) \rightarrow g_{2}(z)$ as $\varepsilon \rightarrow 0$, for all $z \in \mathbb{R}$,

- $\mu_{\varepsilon}(0)=\mu_{\varepsilon}(1)=0$, for all $\varepsilon \in(0,1]$,

- $z \mu_{\varepsilon}(z) \geq 0$, for all $\varepsilon \in(0,1]$,

- there exists a constant $m>0$, independent of $\varepsilon$, such that

$$
\max _{0 \leq z \leq 1} \mu_{\varepsilon}(z)=m \text {. }
$$

For any $\varepsilon \in(0,1]$, let us consider the initial-boundary value problem

$\left(P_{\varepsilon}\right) \quad\left\{\begin{array}{l}f_{\varepsilon t}=\kappa \Delta f_{\varepsilon}+12 L f_{\varepsilon}^{2}\left(1-f_{\varepsilon}\right)-\mu_{\varepsilon}\left(f_{\varepsilon}\right) \frac{\theta_{\varepsilon}}{\theta_{c}} \\ \alpha_{0}\left(\ln \theta_{\varepsilon}\right)_{t}-\frac{1}{\theta_{c}} \mu_{\varepsilon}\left(f_{\varepsilon}\right) f_{\varepsilon t}=k_{0} \Delta \theta_{\varepsilon}+\frac{r}{\theta_{c}}\left(2-\frac{\theta_{\varepsilon}}{\theta_{c}}\right) \\ \left.\nabla f_{\varepsilon} \cdot \mathbf{n}\right|_{\partial \Omega}=0,\left.\quad \theta_{\varepsilon}\right|_{\partial \Omega}=\theta_{\Gamma} \\ f_{\varepsilon}(x, 0)=f_{0}(x), \quad \theta_{\varepsilon}(x, 0)=\theta_{0 \varepsilon}\end{array}\right.$

where the initial data $\theta_{0 \varepsilon}$ are such that

$\theta_{0 \varepsilon} \in H^{1}(\Omega), \quad \theta_{*} \leq \theta_{0 \varepsilon} \leq \theta^{*}$, for any $\varepsilon \in(0,1]$,

$\theta_{0 \varepsilon} \underset{\varepsilon \rightarrow 0}{\rightarrow} \theta_{0}$ in $L^{2}(\Omega)$ and a.e. in $\Omega$.

Thanks to the properties of $\mu_{\varepsilon},\left(P_{\varepsilon}\right)$ is an approximation of the original problem $(P)$ and turns out to be equivalent to $\left(P^{\prime}\right)$ on identifying $\mu_{\varepsilon}$ with $\lambda^{\prime}$. Hence, in view of Theorem 3.1, problem $\left(P_{\varepsilon}\right)$ admits a solution $\left(f_{\varepsilon}, \theta_{\varepsilon}\right)$ satisfying (3.31)-(3.33). Moreover, since $\theta_{\varepsilon}$ is positive a.e., owing to Proposition 2.1 and Remark 2.1 such a solution satisfies

$0 \leq f_{\varepsilon} \leq 1, \quad$ a.e. in $Q_{T}$.

As expected, the existence of solutions $(f, \theta)$ to problem $(P)$ will be achieved by taking any solution $\left(f_{\varepsilon}, \theta_{\varepsilon}\right)$ to problem $\left(P_{\varepsilon}\right)$ and then letting $\varepsilon \rightarrow 0$. In order to prove convergence by means of a compactness argument we need some a priori estimates which ensure the uniform boundedness of solutions to $\left(P_{\varepsilon}\right)$ with respect to $\varepsilon$. The existence result is guaranteed by the following theorem, whose proof will be detailed in further subsections. 
Theorem 3.2. If assumptions (H1)-(H5) are satisfied, then problem $(P)$ admits at least a solution $(f, \theta)$ such that

$f \in L^{2}\left(0, T, H^{2}(\Omega)\right) \cap H^{1}\left(0, T, L^{2}(\Omega)\right)$,

$\theta \in L^{2}\left(0, T, H^{1}(\Omega)\right), \quad \theta>0$ a.e. in $Q_{T}$,

$\ln \theta \in L^{\infty}\left(0, T, L^{2}(\Omega)\right) \cap H^{1}\left(0, T, H^{-1}(\Omega)\right)$.

In addition $0 \leq f \leq 1$ a.e. in $Q_{T}$.

\subsection{Uniform estimates}

Let us multiply the first equation of $\left(P_{\varepsilon}\right)$ by $f_{\varepsilon t}$, the second by $u_{\varepsilon}=\theta_{\varepsilon}-\theta_{\mathcal{H}}$ and integrate over $Q_{t}$. We obtain

$$
\begin{gathered}
\int_{Q_{t}}\left[f_{\varepsilon t}^{2}+k_{0}\left|\nabla u_{\varepsilon}\right|^{2}\right] \mathrm{d} x \mathrm{~d} s+\int_{\Omega}\left[\frac{\kappa}{2}\left|\nabla f_{\varepsilon}(t)\right|^{2}+3 L f_{\varepsilon}^{4}\right. \\
\left.+M_{\varepsilon}\left(f_{\varepsilon}(t)\right) \frac{\theta_{\mathcal{H}}}{\theta_{c}}+\alpha_{0} \theta_{\varepsilon}(t)\right] \mathrm{d} x=I_{1}+I_{2}+I_{3},
\end{gathered}
$$

where

$$
\begin{aligned}
I_{1}= & \int_{\Omega}\left[4 L f_{\varepsilon}^{3}(t)+\alpha_{0} \theta_{\mathcal{H}} \ln \theta_{\varepsilon}(t)\right] \mathrm{d} x, \\
I_{2}= & \int_{Q_{t}} \frac{r}{\theta_{c}}\left(2-\frac{\theta_{\varepsilon}}{\theta_{c}}\right) u_{\varepsilon} \mathrm{d} x \mathrm{~d} s, \\
I_{3}= & \int_{\Omega}\left[\frac{\kappa}{2}\left|\nabla f_{0}\right|^{2}+3 L f_{0}^{4}-4 L f_{0}^{3}+M_{\varepsilon}\left(f_{0}\right) \frac{\theta_{\mathcal{H}}}{\theta_{c}}+\alpha_{0} \theta_{0 \varepsilon}\right. \\
& \left.-\alpha_{0} \theta_{\mathcal{H}} \ln \theta_{0 \varepsilon}\right] \mathrm{d} x,
\end{aligned}
$$

and

$M_{\varepsilon}(z)=\int_{0}^{z} \mu_{\varepsilon}(s) \mathrm{d} s$.

Henceforth we denote by $c$ any positive constant which depends on the structural data of the problem but is independent of $\varepsilon$. It is allowed to take different values even in the same formula. Moreover, in the following estimates we will repeatedly use the Poincaré, Young and Hölder inequalities, jointly with a suitable choice of the constants.

In view of (3.28) and (3.36), we deduce

$I_{1} \leq c+c \int_{\Omega} \ln \theta_{\varepsilon} \mathrm{d} x \leq c+\frac{\alpha_{0}}{2} \int_{\Omega} \theta_{\varepsilon} \mathrm{d} x$.

Moreover,

$I_{2} \leq c\|r\|_{L^{2}\left(Q_{t}\right)}\left\|u_{\varepsilon}\right\|_{L^{2}\left(Q_{t}\right)}-\frac{b}{\theta_{c}^{2}}\left\|u_{\varepsilon}\right\|_{L^{2}\left(Q_{t}\right)}^{2}$,

so that for any $v>0$ we have

$I_{2} \leq c+v\left\|\nabla u_{\varepsilon}\right\|_{L^{2}\left(Q_{t}\right)}^{2}+\max \{0,-b\} \frac{C_{\Omega}}{\theta_{c}^{2}}\left\|\nabla u_{\varepsilon}\right\|_{L^{2}\left(Q_{t}\right)}^{2}$.

Finally, the boundedness of $I_{3}$ follows from assumptions (H2) on the initial data.

Thanks to (H5) and a suitable choice of $v$, substitution in (3.37) yields

$$
\begin{gathered}
\left\|f_{\varepsilon t}\right\|_{L^{2}\left(Q_{t}\right)}^{2}+c\left\|\nabla u_{\varepsilon}\right\|_{L^{2}\left(Q_{t}\right)}^{2}+\frac{\kappa}{2}\left\|\nabla f_{\varepsilon}(t)\right\|^{2} \\
+\int_{\Omega}\left[M_{\varepsilon}\left(f_{\varepsilon}(t)\right) \frac{\theta_{\mathcal{H}}}{\theta_{c}}+\frac{\alpha_{0}}{2} \theta_{\varepsilon}(t)\right] \mathrm{d} x \leq c .
\end{gathered}
$$

Therefore we obtain

$$
\begin{aligned}
& \left\|f_{\varepsilon}\right\|_{L^{\infty}\left(0, T, H^{1}(\Omega)\right) \cap H^{1}\left(0, T, L^{2}(\Omega)\right)}+\left\|u_{\varepsilon}\right\|_{L^{2}\left(0, T, H_{0}^{1}(\Omega)\right)} \\
& \quad+\left\|\theta_{\varepsilon}\right\|_{L^{\infty}\left(0, T, L^{1}(\Omega)\right)} \leq c .
\end{aligned}
$$

By comparison with $\left(P_{\varepsilon}\right)$ and using (3.35), we get

$$
\begin{aligned}
& \left\|\Delta f_{\varepsilon}\right\|_{L^{2}\left(Q_{T}\right)} \leq c, \\
& \left\|\left(\ln \theta_{\varepsilon}\right)_{t}\right\|_{L^{2}\left(0, T, H^{-1}(\Omega)\right)} \leq c .
\end{aligned}
$$

By multiplying the second equation of problem $\left(P_{\varepsilon}\right)$ by $\ln \theta_{\varepsilon}-$ $\ln \theta_{\mathcal{H}}$ and integrating the product over $\Omega$, we achieve

$$
\begin{aligned}
\frac{\alpha_{0}}{2} & \frac{\mathrm{d}}{\mathrm{d} t}\left\|\ln \theta_{\varepsilon}-\ln \theta_{\mathcal{H}}\right\|^{2}+k_{0} \int_{\Omega} \frac{1}{\theta_{\varepsilon}}\left|\nabla \theta_{\varepsilon}\right|^{2} \mathrm{~d} x \\
= & \int_{\Omega}\left[\frac{k_{0}}{\theta_{\mathcal{H}}} \nabla \theta_{\varepsilon} \cdot \nabla \theta_{\mathcal{H}}+\frac{1}{\theta_{c}} \mu\left(f_{\varepsilon}\right) f_{\varepsilon t}\left(\ln \theta_{\varepsilon}-\ln \theta_{\mathcal{H}}\right)\right. \\
& \left.+\frac{r}{\theta_{c}}\left(2-\frac{\theta_{\varepsilon}}{\theta_{c}}\right)\left(\ln \theta_{\varepsilon}-\ln \theta_{\mathcal{H}}\right)\right] \mathrm{d} x .
\end{aligned}
$$

By virtue of (3.35) and (3.38) and assumptions on $\theta_{\mathcal{H}}$, each term on the right-hand side of (3.41) can be estimated by means of the following inequalities:

$$
\begin{aligned}
& \int_{\Omega} \frac{k_{0}}{\theta_{\mathcal{H}}} \nabla \theta_{\varepsilon} \cdot \nabla \theta_{\mathcal{H}} \mathrm{d} x \leq\left\|\nabla \theta_{\varepsilon}\right\|\left\|\nabla \theta_{\mathcal{H}}\right\| \leq\left\|\nabla u_{\varepsilon}\right\|^{2}+c \\
& \int_{\Omega} \frac{1}{\theta_{c}} \mu\left(f_{\varepsilon}\right) f_{\varepsilon t}\left(\ln \theta_{\varepsilon}-\ln \theta_{\mathcal{H}}\right) \mathrm{d} x \leq \frac{m}{\theta_{c}}\left\|f_{\varepsilon t}\right\|\left\|\ln \theta_{\varepsilon}-\ln \theta_{\mathcal{H}}\right\| \\
& \leq \frac{m}{\theta_{c}}\left[\left\|f_{\varepsilon t}\right\|^{2}+\left\|\ln \theta_{\varepsilon}-\ln \theta_{\mathcal{H}}\right\|^{2}\right] \\
& \int_{\Omega} \frac{r}{\theta_{c}}\left(2-\frac{\theta_{\varepsilon}}{\theta_{c}}\right)\left(\ln \theta_{\varepsilon}-\ln \theta_{\mathcal{H}}\right) \mathrm{d} x \\
& \leq c\|r\|^{2}+c\|r\|_{L^{4}(\Omega)}^{2}\left\|\nabla u_{\varepsilon}\right\|^{2}+\left\|\ln \theta_{\varepsilon}-\ln \theta_{\mathcal{H}}\right\|^{2} .
\end{aligned}
$$

Substitution into (3.41) leads to

$$
\begin{aligned}
\frac{\alpha_{0}}{2} & \frac{\mathrm{d}}{\mathrm{d} t}\left\|\ln \theta_{\varepsilon}-\ln \theta_{\mathcal{H}}\right\|^{2}+k_{0} \int_{\Omega} \frac{1}{\theta_{\varepsilon}}\left|\nabla \theta_{\varepsilon}\right|^{2} \mathrm{~d} x \\
\leq & \left(\frac{m}{\theta_{c}}+1\right)\left\|\ln \theta_{\varepsilon}-\ln \theta_{\mathcal{H}}\right\|^{2}+\frac{m}{\theta_{c}}\left\|f_{\varepsilon t}\right\|^{2} \\
& +\left(1+c\|r\|_{L^{4}(\Omega)}^{2}\right)\left\|\nabla u_{\varepsilon}\right\|^{2}+\|r\|^{2}+c .
\end{aligned}
$$

Therefore, keeping (3.38) into account, the Gronwall inequality yields

$\left\|\ln \theta_{\varepsilon}-\ln \theta_{\mathcal{H}}\right\|_{L^{\infty}\left(0, T, L^{2}(\Omega)\right)}^{2} \leq c$,

and finally

$\left\|\ln \theta_{\varepsilon}\right\|_{L^{2}\left(Q_{T}\right)} \leq c$.

\subsection{Proof of Theorem 3.2}

Previous a priori estimates allow us to prove the convergence of any solution $\left(f_{\varepsilon}, \theta_{\varepsilon}\right)$ towards some solution $(f, \theta)$ to problem $(P)$. In view of the uniform bounds (3.38)-(3.40) and (3.42), we deduce, up to subsequences, the following convergences: 
$f_{\varepsilon} \rightarrow f \quad$ weakly in $L^{2}\left(0, T, H^{2}(\Omega)\right)$ and in

$$
H^{1}\left(0, T, L^{2}(\Omega)\right) \text {, }
$$

$u_{\varepsilon} \rightarrow u \quad$ weakly in $L^{2}\left(0, T, H_{0}^{1}(\Omega)\right)$,

$\ln \theta_{\varepsilon} \rightarrow \ell \quad$ weakly in $L^{2}\left(Q_{T}\right)$ and in $H^{1}\left(0, T, H^{-1}(\Omega)\right)$.

Taking (3.30) into account, from (3.44) we easily have

$\theta_{\varepsilon} \rightarrow \theta \quad$ weakly in $L^{2}\left(0, T, H^{1}(\Omega)\right)$.

In addition, the uniform boundedness of the norms

$\left\|f_{\varepsilon}^{2}\left(1-f_{\varepsilon}\right)\right\|_{L^{2}\left(Q_{T}\right)}, \quad\left\|\mu_{\varepsilon}\left(f_{\varepsilon}\right) \theta_{\varepsilon}\right\|_{L^{2}\left(Q_{T}\right)}, \quad\left\|\mu_{\varepsilon}\left(f_{\varepsilon}\right) f_{\varepsilon t}\right\|_{L^{2}\left(Q_{T}\right)}$

ensures their weak convergence in $L^{2}\left(Q_{T}\right)$. Now, in view of the compact embeddings $H^{2}(\Omega) \hookrightarrow H^{1}(\Omega), L^{2}(\Omega) \hookrightarrow H^{-1}(\Omega)$, from (3.43) and (3.45) we deduce

$f_{\varepsilon} \rightarrow f \quad$ strongly in $L^{2}\left(0, T, H^{1}(\Omega)\right)$,

$\ln \theta_{\varepsilon} \rightarrow \ell \quad$ strongly in $L^{2}\left(0, T, H^{-1}(\Omega)\right)$,

so that (see [19, p.12], for instance)

$12 L f_{\varepsilon}^{2}\left(1-f_{\varepsilon}\right) \rightarrow 12 L f^{2}(1-f)=g_{2}(f)$

weakly in $L^{2}\left(Q_{T}\right)$.

The inequality

$$
\begin{aligned}
\left|\mu_{\varepsilon}\left(f_{\varepsilon}\right)-g_{2}\left(f_{\varepsilon}\right)\right| & \leq\left|\mu_{\varepsilon}\left(f_{\varepsilon}\right)-\mu_{\varepsilon}(f)\right|+\left|\mu_{\varepsilon}(f)-g_{2}(f)\right| \\
& \leq c\left|f_{\varepsilon}-f\right|+\left|\mu_{\varepsilon}(f)-g_{2}(f)\right|,
\end{aligned}
$$

jointly with previous results, provides

$\mu_{\varepsilon}\left(f_{\varepsilon}\right) \rightarrow g_{2}(f) \quad$ strongly in $L^{2}\left(Q_{T}\right)$

and thanks to (3.43) and (3.46) we obtain in addition

$\mu_{\varepsilon}\left(f_{\varepsilon}\right) \theta_{\varepsilon} \rightarrow f^{2}(1-f) \theta \quad$ weakly in $L^{2}\left(Q_{T}\right)$,

$\mu_{\varepsilon}\left(f_{\varepsilon}\right) f_{\varepsilon t} \rightarrow f^{2}(1-f) f_{t} \quad$ weakly in $L^{2}\left(Q_{T}\right)$.

Concerning the logarithmic term, we notice that

$$
\begin{aligned}
\int_{Q_{T}} \theta_{\varepsilon} \ln \theta_{\varepsilon} \mathrm{d} x \mathrm{~d} t= & \int_{Q_{T}}\left(u_{\varepsilon}+\theta_{\mathcal{H}}\right) \ln \theta_{\varepsilon} \mathrm{d} x \mathrm{~d} t \rightarrow\langle\langle\ell, u\rangle\rangle \\
& +\int_{Q_{T}} \theta_{\mathcal{H}} \ell \mathrm{d} x \mathrm{~d} t
\end{aligned}
$$

By applying [1, p.42], we prove $\ell=\ln \theta$ and then $\theta>0$ a.e. in $Q_{T}$.

The final part of Theorem 3.2 follows from Proposition 2.1.

\section{Uniqueness of the solution}

In this section we prove the uniqueness of the solution to problem $(P)$. The following theorem shows the continuous dependence on the data, which ensures the uniqueness of the solution. For every function $\varphi$ of $x, t$ we define

$\hat{\varphi}(x, t)=\int_{0}^{t} \varphi(x, s) \mathrm{d} s$.
Theorem 4.1. If $\left(f_{1}, \theta_{1}\right),\left(f_{2}, \theta_{2}\right)$ are two solutions to problem $(P)$ with $f_{01}, \theta_{01}, \theta_{\Gamma 1}, r_{1}$ and $f_{02}, \theta_{02}, \theta_{\Gamma 2}, r_{2}$ satisfying (H1)-(H5), then

$$
\begin{aligned}
& \| f_{1}(t)-f_{2}(t)\left\|^{2}+\right\| \nabla f_{1}-\nabla f_{2} \|_{L^{2}\left(Q_{t}\right)}^{2} \\
&+\int_{Q_{t}}\left(\ln \theta_{1}-\ln \theta_{2}\right)\left(\theta_{1}-\theta_{2}\right) \mathrm{d} x \mathrm{~d} s \\
&+\left\|\nabla \hat{u}_{1}(t)-\nabla \hat{u}_{2}(t)\right\|^{2} \\
& \leq M\left(\left\|f_{01}-f_{02}\right\|^{2}+\left\|r_{1}-r_{2}\right\|_{L^{2}\left(Q_{T}\right)}^{2}\right. \\
& \quad+\left\|F_{01}-F_{02}\right\|^{2}+\left\|\theta_{\Gamma 1}-\theta_{\Gamma 2}\right\|_{H^{1 / 2}(\partial \Omega)} \\
&\left.\quad+\left\|\theta_{\Gamma 1}-\theta_{\Gamma 2}\right\|_{H^{1 / 2}(\partial \Omega)}^{2}\right),
\end{aligned}
$$

where

$F_{0 i}=\alpha_{0} \ln \theta_{0 i}-\frac{12 L}{\theta_{c}}\left[\frac{1}{4} f_{0 i}^{4}-\frac{2}{3} f_{0 i}^{3}+\frac{1}{2} f_{0 i}^{2}\right], \quad i=1,2$

and $M$ is a constant depending on $f_{0 i}, \theta_{0 i}, \theta_{\Gamma i}, r_{i}, i=1,2$.

Proof. Let $\left(f_{i}, \theta_{i}\right), i=1,2$, be two solutions to $(P)$, and let

$$
\begin{aligned}
& f=f_{1}-f_{2}, \quad \theta=\theta_{1}-\theta_{2}, \quad r=r_{1}-r_{2}, \\
& F_{0}=F_{01}-F_{02}, \quad \theta_{\Gamma}=\theta_{\Gamma 1}-\theta_{\Gamma 2}, \quad \theta_{\mathcal{H}}=\theta_{\mathcal{H} 1}-\theta_{\mathcal{H} 2} .
\end{aligned}
$$

By integrating the second equation of $(P)$ over the time interval $(0, t)$, for each $i=1,2$ we obtain

$$
\begin{aligned}
\alpha_{0} \ln \theta_{i}-k_{0} \Delta \hat{\theta}_{i}= & \frac{12 L}{\theta_{c}}\left[\frac{1}{4} f_{i}^{4}-\frac{2}{3} f_{i}^{3}+\frac{1}{2} f_{i}^{2}\right] \\
& +\frac{1}{\theta_{c}} \int_{0}^{t} r_{i}\left(2-\frac{\theta_{i}}{\theta_{c}}\right) \mathrm{d} s+F_{0 i}
\end{aligned}
$$

and upon subtracting them it follows that

$$
\begin{aligned}
& \alpha_{0}\left(\ln \theta_{1}-\ln \theta_{2}\right)-k_{0} \Delta \hat{\theta} \\
& =\frac{12 L}{\theta_{c}}\left[\frac{1}{4}\left(f_{1}^{4}-f_{2}^{4}\right)-\frac{2}{3}\left(f_{1}^{3}-f_{2}^{3}\right)+\frac{1}{2}\left(f_{1}^{2}-f_{2}^{2}\right)\right] \\
& \quad+\frac{2}{\theta_{c}} \hat{r}-\frac{1}{\theta_{c}^{2}} \int_{0}^{t}\left(r_{1} \theta_{1}-r_{2} \theta_{2}\right) \mathrm{d} s+F_{0} .
\end{aligned}
$$

In a similar way the first equation of $(P)$ yields

$$
\begin{aligned}
f_{t}-\kappa \Delta f= & 12 L\left[f_{1}^{2}\left(1-f_{1}\right)-f_{2}^{2}\left(1-f_{2}\right)\right] \\
& -\frac{12 L}{\theta_{c}}\left[\theta_{1} f_{1}\left(1-f_{1}\right)^{2}-\theta_{2} f_{2}\left(1-f_{2}\right)^{2}\right] .
\end{aligned}
$$

Let us multiply (4.50) by $f$ and (4.49) by $u=u_{1}-u_{2}=\theta-\theta_{\mathcal{H}}$. By integrating over $Q_{t}$ and adding the resulting equations, we obtain

$$
\begin{aligned}
\frac{1}{2}\|f(t)\|^{2}+\kappa\|\nabla f\|_{L^{2}\left(Q_{t}\right)}^{2}+\alpha_{0} \int_{Q_{t}}\left(\ln \theta_{1}-\ln \theta_{2}\right) \theta \mathrm{d} x \mathrm{~d} s \\
\quad+\frac{k_{0}}{2}\|\nabla \hat{u}(t)\|^{2} \\
=\frac{1}{2}\left\|f_{01}-f_{02}\right\|^{2}+J_{1}+J_{2}+J_{3}+J_{4},
\end{aligned}
$$


where

$$
\begin{aligned}
J_{1}= & 12 L \int_{Q_{t}}\left[f_{1}^{2}\left(1-f_{1}\right)-f_{2}^{2}\left(1-f_{2}\right)-\frac{\theta_{\mathcal{H} 1}}{\theta_{c}} f_{1}\left(1-f_{1}\right)^{2}\right. \\
& \left.+\frac{\theta_{\mathcal{H} 2}}{\theta_{c}} f_{2}\left(1-f_{2}\right)^{2}\right] f \mathrm{~d} x \mathrm{~d} s \\
J_{2}= & \frac{12 L}{\theta_{c}} \int_{Q_{t}}\left\{\left[\frac{1}{4}\left(f_{1}^{4}-f_{2}^{4}\right)-\frac{2}{3}\left(f_{1}^{3}-f_{2}^{3}\right)\right.\right. \\
& \left.+\frac{1}{2}\left(f_{1}^{2}-f_{2}^{2}\right)\right] u-\left[u_{1} f_{1}\left(1-f_{1}\right)^{2}\right. \\
& \left.\left.-u_{2} f_{2}\left(1-f_{2}\right)^{2}\right] f\right\} \mathrm{d} x \mathrm{~d} s \\
J_{3}= & \int_{Q_{t}}\left[\frac{2}{\theta_{c}} \hat{r}-\frac{1}{\theta_{c}^{2}} \int_{0}^{s}\left(r_{1} \theta_{1}-r_{2} \theta_{2}\right) \mathrm{d} \tau+F_{0}\right] u \mathrm{~d} x \mathrm{~d} s \\
J_{4}= & \alpha_{0} \int_{Q_{t}}\left(\ln \theta_{1}-\ln \theta_{2}\right) \theta_{\mathcal{H}} \mathrm{d} x \mathrm{~d} s .
\end{aligned}
$$

Now we estimate individually each integral $J_{1}, \ldots, J_{4}$. We have

$$
\begin{aligned}
J_{1}= & 12 L \int_{Q_{t}}\left[\left(f_{1}+f_{2}\right) f^{2}-\left(f_{1}^{2}+f_{1} f_{2}+f_{2}^{2}\right) f^{2}\right. \\
& -\frac{1}{\theta_{c}} f_{1}\left(1-f_{1}\right)^{2} \theta_{\mathcal{H}} f-\frac{\theta_{\mathcal{H} 2}}{\theta_{c}} f^{2}\left(1+f_{1}^{2}+f_{1} f_{2}+f_{2}^{2}\right) \\
& \left.+\frac{2 \theta_{\mathcal{H} 2}}{\theta_{c}}\left(f_{1}+f_{2}\right) f^{2}\right] \mathrm{d} x \mathrm{~d} s \\
\leq & 12 L \int_{Q_{t}}\left[\left(f_{1}+f_{2}\right) f^{2}-\frac{1}{\theta_{c}} f_{1}\left(1-f_{1}\right)^{2} \theta_{\mathcal{H}} f\right. \\
& \left.+\frac{2 \theta_{\mathcal{H} 2}}{\theta_{c}}\left(f_{1}+f_{2}\right) f^{2}\right] \mathrm{d} x \mathrm{~d} s .
\end{aligned}
$$

In view of Proposition 2.1, we obtain

$J_{1} \leq c\left\{\|f\|_{L^{2}\left(Q_{t}\right)}^{2}+\int_{0}^{t}\left[\left\|\theta_{\mathcal{H}}\right\|+\|f(s)\|_{H^{1}(\Omega)}\right]\|f(s)\| \mathrm{d} s\right\}$.

Therefore, owing to (3.30), for any $v>0$ it follows that

$J_{1} \leq c\|f\|_{L^{2}\left(Q_{t}\right)}^{2}+v\|\nabla f\|_{L^{2}\left(Q_{t}\right)}^{2}+c\left\|\theta_{\Gamma}\right\|_{H^{1 / 2}(\partial \Omega)}^{2}$.

By rearranging the terms of $J_{2}$, we obtain

$$
\begin{aligned}
J_{2}= & \frac{12 L}{\theta_{c}} \int_{Q_{t}}\left\{-u_{1}\left[\frac{3}{4} f_{1}^{2}+\frac{1}{2} f_{1} f_{2}+\frac{1}{4} f_{2}^{2}-\frac{4}{3} f_{1}-\frac{2}{3} f_{2}\right.\right. \\
& \left.+\frac{1}{2}\right]+u_{2}\left[\frac{3}{4} f_{2}^{2}+\frac{1}{2} f_{1} f_{2}+\frac{1}{4} f_{1}^{2}-\frac{4}{3} f_{2}-\frac{2}{3} f_{1}\right. \\
& \left.\left.+\frac{1}{2}\right]\right\} f^{2} \mathrm{~d} x \mathrm{~d} s .
\end{aligned}
$$

Hence, in view of the boundedness of $f_{1}, f_{2}$, we deduce

$$
\begin{aligned}
J_{2} \leq & c \int_{Q_{t}}\left(\left|u_{1}\right|+\left|u_{2}\right|\right) f^{2} \mathrm{~d} x \mathrm{~d} s \\
\leq & c \int_{0}^{t}\left(\left\|u_{1}(s)\right\|_{L^{4}(\Omega)}+\left\|u_{2}(s)\right\|_{L^{4}(\Omega)}\right)\|f(s)\|_{L^{4}(\Omega)} \\
& \times\|f(s)\| \mathrm{d} s
\end{aligned}
$$

$$
\begin{aligned}
\leq & v\|\nabla f\|_{L^{2}\left(Q_{t}\right)}^{2}+c \int_{0}^{t}\left(\left\|u_{1}(s)\right\|_{H^{1}(\Omega)}^{2}+\left\|u_{2}(s)\right\|_{H^{1}(\Omega)}^{2}\right) \\
& \times\|f(s)\|^{2} \mathrm{~d} s .
\end{aligned}
$$

Let us examine the third integral $J_{3}$. An integration by parts yields

$$
\begin{aligned}
J_{3}= & \int_{\Omega}\left[\frac{2}{\theta_{c}} \hat{r}(t)-\frac{1}{\theta_{c}^{2}} \int_{0}^{t}\left(r_{1} \theta_{1}-r_{2} \theta_{2}\right) \mathrm{d} \tau+F_{0}\right] \hat{u}(t) \mathrm{d} x \\
& -\int_{Q_{t}}\left[\frac{2}{\theta_{c}} r-\frac{1}{\theta_{c}^{2}}\left(r_{1} \theta_{1}-r_{2} \theta_{2}\right)\right] \hat{u} \mathrm{~d} x \mathrm{~d} s .
\end{aligned}
$$

Hence

$$
\begin{aligned}
J_{3} \leq & c\left[\|\hat{r}(t)\|+\left\|r \theta_{1}\right\|_{L^{2}\left(Q_{t}\right)}+\left\|r_{2} \theta \mathcal{H}\right\|_{L^{2}\left(Q_{t}\right)}+\left\|F_{0}\right\|\right]\|\hat{u}(t)\| \\
& +c\left[\|r\|_{L^{2}\left(Q_{t}\right)}+\left\|r \theta_{1}\right\|_{L^{2}\left(Q_{t}\right)}+\left\|r_{2} \theta_{\mathcal{H}}\right\|_{L^{2}\left(Q_{t}\right)}\right]\|\hat{u}\|_{L^{2}\left(Q_{t}\right)} \\
& -\frac{1}{\theta_{c}^{2}} \int_{\Omega}\left[\int_{0}^{t} r_{2} u \mathrm{~d} s \hat{u}(t)-\int_{0}^{t} r_{2} u \hat{u} \mathrm{~d} s\right] \mathrm{d} x .
\end{aligned}
$$

A further integration by parts leads to the relation

$$
\begin{aligned}
- & \frac{1}{\theta_{c}^{2}} \int_{\Omega}\left[\hat{u}(t) \int_{0}^{t} r_{2} u \mathrm{~d} s-\int_{0}^{t} r_{2} u \hat{u} \mathrm{~d} s\right] \mathrm{d} x \\
= & -\frac{1}{\theta_{c}^{2}} \int_{\Omega}\left[\frac{1}{2} r_{2}(t) \hat{u}(t)-\hat{u}(t) \int_{0}^{t} r_{2 t} \hat{u} \mathrm{~d} s+\int_{0}^{t} r_{2 t} \hat{u}^{2} \mathrm{~d} s\right] \mathrm{d} x \\
\leq & -\frac{b}{2 \theta_{c}^{2}}\|\hat{u}(t)\|^{2}+c\left\|r_{2 t}\right\|_{L^{\infty}\left(Q_{t}\right)}\left(\|\hat{u}(t)\|\|\hat{u}\|_{L^{2}\left(Q_{t}\right)}\right. \\
& \left.+\|\hat{u}\|_{L^{2}\left(Q_{t}\right)}^{2}\right) .
\end{aligned}
$$

Thus, by means of the Young and Poincaré inequalities we obtain

$$
\begin{aligned}
J_{3} \leq & v\|\nabla \hat{u}(t)\|^{2}+c\left(1+\left\|r_{2 t}\right\|_{L^{\infty}\left(Q_{t}\right)}\right)\|\nabla \hat{u}\|_{L^{2}\left(Q_{t}\right)}^{2} \\
& +c\left(\|r\|_{L^{2}\left(Q_{t}\right)}^{2}+\|r\|_{L^{\infty}\left(Q_{t}\right)}^{2}\left\|\theta_{1}\right\|_{L^{2}\left(Q_{t}\right)}^{2}\right. \\
& \left.+\left\|r_{2}\right\|_{L^{\infty}\left(Q_{t}\right)}^{2}\left\|\theta_{\mathcal{H}}\right\|_{L^{2}\left(Q_{t}\right)}^{2}+\left\|F_{0}\right\|^{2}\right)+\max \{0,-b\} \frac{C_{\Omega}}{2 \theta_{c}^{2}} \\
& \times\|\nabla \hat{u}(t)\|^{2} .
\end{aligned}
$$

Finally, the last integral $J_{4}$ can be estimated as $J_{4} \leq \alpha_{0}\left(\left\|\ln \theta_{1}\right\|_{L^{1}\left(0, T, L^{2}(\Omega)\right)}+\left\|\ln \theta_{2}\right\|_{L^{1}\left(0, T, L^{2}(\Omega)\right)}\right)\left\|\theta_{\mathcal{H}}\right\|$.

Substitution of (4.52)-(4.55) into (4.51) and a suitable choice of $v$ lead to the inequality

$$
\begin{aligned}
& \frac{1}{2}\|f(t)\|^{2}+\frac{\kappa}{2}\|\nabla f\|_{L^{2}\left(Q_{t}\right)}^{2}+\alpha_{0} \int_{Q_{t}}\left(\ln \theta_{1}-\ln \theta_{2}\right) \theta \mathrm{d} x \mathrm{~d} s \\
& \quad+c\|\nabla \hat{u}(t)\|^{2} \leq \int_{0}^{t} \varphi_{1}(s)\left[\|f(s)\|^{2}+\|\nabla \hat{u}(s)\|^{2}\right] \mathrm{d} x \mathrm{~d} s \\
& \quad+\varphi_{2}(t),
\end{aligned}
$$

where

$$
\begin{aligned}
\varphi_{1}(t)= & c\left[1+\left\|u_{1}(t)\right\|_{H^{1}(\Omega)}^{2}+\left\|u_{2}(t)\right\|_{H^{1}(\Omega)}^{2}+\left\|r_{2 t}\right\|_{L^{\infty}\left(Q_{t}\right)}\right] \\
\varphi_{2}(t)= & c\left[\left\|f_{0}\right\|^{2}+\|r\|_{L^{2}\left(Q_{t}\right)}^{2}+\|r\|_{L^{\infty}\left(Q_{t}\right)}^{2}\left\|\theta_{1}\right\|_{L^{2}\left(Q_{t}\right)}^{2}\right. \\
& \left.+\left\|F_{0}\right\|^{2}+\left\|\theta_{\Gamma}\right\|_{H^{1 / 2}(\partial \Omega)}^{2}+\left\|\theta_{\Gamma}\right\|_{H^{1 / 2}(\partial \Omega)}\right] .
\end{aligned}
$$


Therefore, the Gronwall inequality provides

$$
\begin{aligned}
& \|f(t)\|^{2}+\|\nabla f\|_{L^{2}\left(Q_{t}\right)}^{2}+\int_{Q_{t}}\left(\ln \theta_{1}-\ln \theta_{2}\right) \theta \mathrm{d} x \mathrm{~d} s \\
& +\|\nabla \hat{u}(t)\|^{2} \leq \int_{0}^{t} \varphi_{1}(s) \varphi_{2}(s) \mathrm{e}^{\int_{s}^{t} \varphi_{1}(\tau) \mathrm{d} \tau} \mathrm{d} s+\varphi_{2}(t),
\end{aligned}
$$

so (4.47) holds.

\section{References}

[1] V. Barbu, Nonlinear Semigroups and Differential Equations in Banach Spaces, Noordhoff International Publishing, Leiden, 1976.

[2] E. Bonetti, P. Colli, M. Fabrizio, G. Gilardi, Global solution to a singular integrodifferential system related to the entropy balance, Nonlinear Anal. TMA 66 (2007) 1949-1979.

[3] E. Bonetti, P. Colli, M. Fabrizio, G. Gilardi, Modelling and long-time behaviour for phase transitions with entropy balance and thermal memory conductivity, Discrete Contin. Dyn. Syst. Ser. B 6 (2006) 1001-1026.

[4] E. Bonetti, P. Colli, M. Frémond, A phase field model with thermal memory governed by the entropy balance, Math. Models Methods Appl. Sci. 13 (2003) 1565-1588.

[5] H. Brezis, Operateurs maximaux monotones et semi-groupes de contractions dans les espaces de Hilbert, in: North-Holland Math. Stud., vol. 5, North-Holland, Amsterdam, 1973.

[6] M. Brokate, J. Sprekels, Hysteresis and Phase Transitions, Springer, New York, 1996.

[7] G. Caginalp, An analysis of a phase field model of a free boundary, Arch. Ration. Mech. Anal. 92 (1986) 205-245.

[8] P. Colli, G. Gentili, C. Giorgi, Nonlinear systems describing phase transition models compatible with thermodynamics, Math. Models Methods Appl. Sci. 9 (1999) 1015-1037.

[9] M. Fabrizio, Ginzburg-Landau equations and first and second order phase transitions, Internat. J. Engrg. Sci. 44 (2006) 529-539.
[10] M. Fabrizio, C. Giorgi, A. Morro, A thermodynamic approach to nonisothermal phase-field evolution in continuum physics, Physica D 214 (2006) 144-156

[11] M. Frémond, Non-Smooth Thermomechanics, Springer-Verlag, Berlin, 2002.

[12] H. Garcke, B. Nestler, B. Stinner, A diffusive interface model for alloys with multiple components and phases, SIAM J. Appl. Math. 64 (2004) 775-799.

[13] V.L. Ginzburg, L.D. Landau, On the theory of superconductivity, Zh. Eksp. Teor. Fiz. 20 (1950) 1064-1072.

[14] A. Karma, W.J. Rappel, Quantitative phase-field modeling of dendritic growth in two and three dimensions, Phys. Rev. E 57 (1998) 4323-4349.

[15] N. Kenmochi, Attractors of semigroups associated with nonlinear systems for diffusive phase separation, Abstr. Appl. Anal. 1 (1996) 169-192.

[16] N. Kenmochi, M. Niezgódka, Evolution systems of nonlinear variational inequalities arising phase change problems, Nonlinear Anal. 22 (1994) 1163-1180.

[17] C. Kittel, Introduction to Solid State Physics, J. Wiley \& Sons, New York, 1968.

[18] P. Laurencot, Long-time behaviour for a model of phase-field type, Proc. Roy. Soc. Edinburgh Sect. A 126 (1996) 167-185.

[19] J.L. Lions, Quelques methodes de resolution des problemes aux limites non lineaires, Dunod-Gauthier Villars, Paris, 1969.

[20] O. Penrose, P.C. Fife, On the relation between the standard phase-field model and a "thermodynamically consistent" phase-field model, Physica D 69 (1993) 107-113.

[21] J. Sprekels, S. Zheng, Maximal attractor for the system of a Landau-Ginzburg theory for structural phase transitions in shape memory alloys, Physica D 121 (1998) 252-262.

[22] S.L. Wang, R.F. Sekerka, A.A. Wheeler, B.T. Murray, S.R. Coriell, R.J. Braun, G.B. McFadden, Thermodynamically consistent phase-field models for solidification, Physica D 69 (1993) 189-200.

[23] A.A. Wheeler, G.B. McFadden, A $\xi$-vector formulation of anisotropic phase-field models: 3D asymptotics, European J. Appl. Math. (1996) $367-381$. 